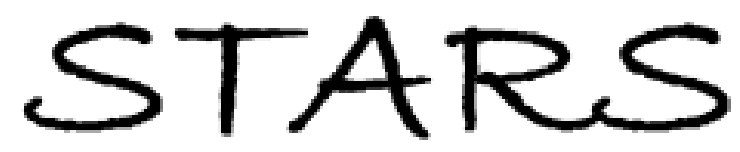

University of Central Florida

STARS

12-31-1999

\title{
Scintillation model for a satellite communication link at large zenith angles
}

\author{
Larry C. Andrews \\ University of Central Florida \\ Ronald L. Phillips \\ University of Central Florida \\ Cynthia Young Hopen \\ University of Central Florida
}

Find similar works at: https://stars.library.ucf.edu/facultybib2000

University of Central Florida Libraries http://library.ucf.edu

This Article is brought to you for free and open access by the Faculty Bibliography at STARS. It has been accepted for inclusion in Faculty Bibliography 2000 s by an authorized administrator of STARS. For more information, please contact STARS@ucf.edu.

\section{Recommended Citation}

Andrews, Larry C.; Phillips, Ronald L.; and Hopen, Cynthia Young, "Scintillation model for a satellite communication link at large zenith angles" (1999). Faculty Bibliography 2000s. 2424.

https://stars.library.ucf.edu/facultybib2000/2424

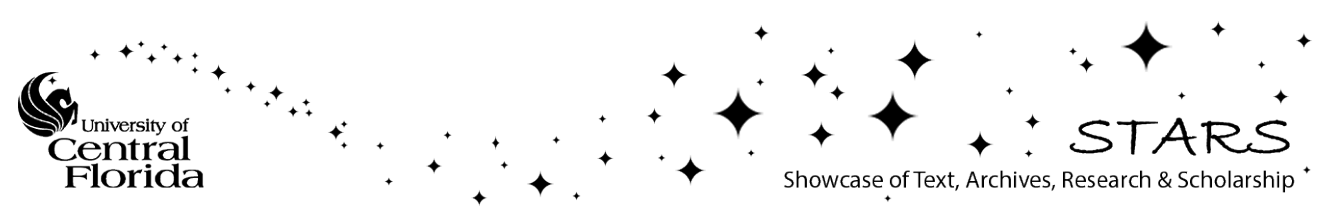




\section{Scintillation model for a satellite communication link at large zenith angles}

\author{
Larry C. Andrews \\ University of Central Florida \\ Department of Mathematics and School of \\ Optics/CREOL \\ Orlando, Florida 32816 \\ E-mail: landrews@pegasus.cc.ucf.edu \\ Ronald L. Phillips \\ University of Central Florida \\ Department of Electrical and Computer \\ Engineering \\ and \\ Florida Space Institute \\ Orlando, Florida 32816 \\ Cynthia Young Hopen \\ University of Central Florida \\ Department of Mathematics \\ and \\ Florida Space Institute \\ Orlando, Florida 32816
}

\begin{abstract}
A scintillation model is developed for uplink-downlink optical communication channels applicable in moderate to strong fluctuation conditions that may arise under large zenith angles between transmitter and receiver. The model developed here is an extension of a recently published theory that treats irradiance fluctuations along a horizontal path as a modulation of small-scale scintillation by large-scale scintillation. For a downlink path the scintillation index is modeled like that of an infinite plane wave, and for an uplink path we consider a spherical wave model. In both cases the scintillation index agrees with conventional weak-fluctuation-theory results out to zenith angles of 45 to $60 \mathrm{deg}$. The covariance function of irradiance fluctuations is also developed under the same conditions as assumed for the scintillation index. On a downlink path under small zenith angles the implied correlation length is proportional to the Fresnel-zone scale. For zenith angles exceeding $85 \mathrm{deg}$, the downlink correlation length varies directly with the spatial coherence radius weighted by a factor that depends on changes in $C_{n}^{2}$ the refractive index structural parameter with altitude. (c) 2000 Society of Photo-Optical Instrumentation Engineers. [S0091-3286(00)02412-0]
\end{abstract}

Subject terms: atmospheric optics; scintillation; optical wave propagation; laser satellite communication.

Paper 990315 received Aug. 10, 1999; revised manuscript received July 7, 2000; accepted for publication July 28, 2000.

\section{Introduction}

For more than three decades the scientific community has expressed interest in the possibility of using high-data-rate optical transmitters for satellite communications. ${ }^{1-12}$ This interest stems from the advantages offered by optical wave systems over conventional rf systems, such as smaller antennas; less mass, power, and volume; and the intrinsic narrow beam and high gain of lasers. Developmental programs in the USA involving optical communications started in the 1960s with the development of a $\mathrm{CO}_{2}$ system by NASA and a Nd:YAG direct detection system by the Air Force. In the 1970s and 1980s there were a number of follow-on developmental programs like the Space Flight Test System (SFTS), Airborne Flight Test System (AFTS), Laser Crosslink Subsystem (LCS), and Follow-on Early Warning System (FEWS). Unfortunately, most of these government developmental programs were terminated due to funding cutbacks. ${ }^{1}$

During the 1990s there has been renewed interest in the use of high-data-rate optical transmitters for satellite communication channels connecting ground/airborne-to-space or space-to-ground/airborne data links. Although optical communication systems offer several advantages as stated above, laser-satellite communication systems are subject to severe signal fading below a prescribed threshold value, owing primarily to optical scintillations associated with the received signal. Several papers have dealt with actual measurements of atmospherically induced scintillation and/or the development of theoretical models for predicting scintillation levels. ${ }^{3-12}$ However, the previously developed the- oretical models are all based on conditions of weak fluctuations, which generally limits the assumed zenith angle to 45 to $60 \mathrm{deg}$ or less. For greater zenith angles, moderate to strong-fluctuation theory must generally be used to predict optical scintillation, but thus far no tractable models have been developed for this case.

A heuristic scintillation model was introduced recently that is applicable under moderate to strong irradiance fluctuations, but limited to atmospheric channels along horizontal paths in which the refractive-index structure parameter $C_{n}^{2}$ is assumed to be constant. ${ }^{13}$ This new model is based on a modulation process in which small-scale scintillation is modulated by large-scale scintillation. In particular, it takes into account the continued loss of transverse spatial coherence of the optical wave as it propagates from weak to strong fluctuation regimes. The loss of spatial coherence is allowed for by the formal introduction of a spatial amplitude filter that eliminates ineffective cell sizes between the correlation length and scattering disk in multiplescattering regimes. In this paper we build upon that model by introducing a modification of the filter function to account for changes in $C_{n}^{2}$ as a function of propagation distance. Our analysis includes models for scintillation and the covariance function of irradiance fluctuations on both uplink and downlink laser-satellite communication channels.

\section{The Modulation Process}

In this section we first briefly review the theory of scintillation developed in Ref. 13 for horizontal paths with con- 
stant refractive index structure parameter. Building on that theory, we then extend the results to vertical or slant paths.

To begin, we assume the received irradiance of the optical wave can be modeled as a modulation process in which small-scale (diffractive) fluctuations are multiplicatively modulated by large-scale (refractive) fluctuations. Thus, similarly to previous treatments, ${ }^{14-16}$ the received irradiance can be expressed as a product $I=x y$, where $x$ arises from large-scale turbulent cells, or eddies, and $y$ from small-scale cells. If we further assume that $x$ and $y$ are statistically independent and that $\langle I\rangle=1$, the scintillation index $\sigma_{I}^{2}=\left\langle I^{2}\right\rangle /\langle I\rangle^{2}-1$ takes the form

$\sigma_{I}^{2}=\left\langle x^{2}\right\rangle\left\langle y^{2}\right\rangle-1=\left(1+\sigma_{x}^{2}\right)\left(1+\sigma_{y}^{2}\right)-1=\sigma_{x}^{2}+\sigma_{y}^{2}+\sigma_{x}^{2} \sigma_{y}^{2}$,

where \langle\rangle denotes an ensemble average and where $\sigma_{x}^{2}$ and $\sigma_{y}^{2}$ are the variances of $x$ and $y$, respectively. Moreover, we can express $\sigma_{x}^{2}$ and $\sigma_{y}^{2}$ in terms of log-irradiance variances of $x$ and $y$ according to ${ }^{13} \sigma_{x}^{2}=\exp \left(\sigma_{\ln x}^{2}\right)-1$ and $\sigma_{y}^{2}$ $=\exp \left(\sigma_{\ln y}^{2}\right)-1$, which permits us to rewrite Eq. (1) equivalently as

$\sigma_{I}^{2}=\exp \left(\sigma_{\ln x}^{2}+\sigma_{\ln y}^{2}\right)-1$

Small-scale scintillation is caused by diffractive turbulence cells on the order of the correlation length of the irradiance fluctuations, and large-scale scintillation is caused by refractive cells on the order of the scattering disk. The scattering disk is defined by the refractive cell size $l$ at which the focusing angle $\theta_{F} \sim l / L$ is equal to the average diffraction angle $\theta_{D}$, where $\theta_{D} \sim 1 / \sqrt{k L}$ in weak fluctuations and $\theta_{D} \sim 1 / k \rho_{0}$ in strong fluctuations. The correlation length and scattering disk of an optical wave in weak irradiance fluctuations are both on the order of the first Fresnel zone $(L / k)^{1 / 2}$, whereas in strong fluctuations the correlation length is on the order of the transverse spatial coherence radius $\rho_{0}$ and the scattering disk is characterized by $L / k \rho_{0}$. The parameter $L$ represents total path length between transmitter and receiver, and $k$ is the optical wave number.

\subsection{Spatial Filter Function: Constant- $C_{n}^{2}$ Model}

The assumed model for the power spectrum of refractive index fluctuations is the conventional Kolmogorov spectrum $\Phi_{n}(\kappa, z)=0.033 C_{n}^{2}(z) \kappa^{-11 / 3}$, where $z$ represents the propagation distance from the transmitter. Because of the assumed modulation process, we formally replace $\Phi_{n}(\kappa, z)$ with the effective spectrum model

$\Phi_{n, e}(\kappa, z)=0.033 C_{n}^{2}(z) \kappa^{-11 / 3} G(\kappa, z)$,

where $G(\kappa, z)$ is a spatial filter induced by the propagation process and $z$ is a propagation distance that varies between $z=0$ and $z=L$. For propagation environments where $C_{n}^{2}$ is essentially constant, the induced filter function can be approximated by ${ }^{13}$
$G(\kappa, z) \approx G_{x}(\kappa)+G_{y}(\kappa)=\exp \left(-\frac{\kappa^{2}}{\kappa_{x}^{2}}\right)+\frac{\kappa^{11 / 3}}{\left(\kappa^{2}+\kappa_{y}^{2}\right)^{11 / 6}}$,

in which there is no explicit dependence on the propagation variable $z$. (Inner-scale effects were taken into account in Ref. 13 but are neglected in the present analysis.) The quantity $G_{x}(\kappa)$ is the large-scale filter function, and $G_{y}(\kappa)$ is the small-scale filter function, both of which are selected on the basis of mathematical tractability. Other functional forms for these filter functions may be just as appropriate and can lead to similar results to those presented below. The parameter $\kappa_{x}$ is a large-scale (or refractive) spatialfrequency cutoff much like an inner-scale parameter, and $\kappa_{y}$ is a small-scale (or diffractive) spatial-frequency cutoff similar to an outer-scale parameter. In this fashion, $G(\kappa, z)$ acts like an amplitude (or irradiance) spatial filter function that only permits low-pass spatial frequencies $\kappa<\kappa_{x}$ and high-pass spatial frequencies $\kappa>\kappa_{y}$ at a given propagation distance.

The low-pass and high-pass spatial-frequency cutoffs appearing in the filter function (4) are directly related to the correlation length and scattering disk of the fluctuating irradiance. Hence, at any distance $L$ into the random medium, we assume the existence of an effective scattering disk $L / k l_{x}$ and an effective correlation length $l_{y}$ related, respectively, to the cutoff wave numbers according to

$\frac{L}{k l_{x}}=\frac{1}{\kappa_{x}} \sim \begin{cases}\sqrt{L / k}, & L / k \rho_{0}^{2} \ll 1, \\ L / k \rho_{0}, & L / k \rho_{0}^{2} \gg 1,\end{cases}$
$l_{y}=\frac{1}{\kappa_{y}} \sim \begin{cases}\sqrt{L / k}, & L / k \rho_{0}^{2} \ll 1, \\ \rho_{0}, & L / k \rho_{0}^{2} \gg 1 .\end{cases}$

Here, $L / k \rho_{0}^{2} \ll 1$ represents weak fluctuation conditions and $L / k \rho_{0}^{2} \gg 1$ represents strong fluctuations when $C_{n}^{2}$ is constant.

\subsection{Spatial Filter Function: Variable- $C_{n}^{2}$ Model}

Propagation along a vertical or slant path requires a $C_{n}^{2}(h)$ profile model to describe properly the varying strength of optical turbulence as a function of altitude $h$. One of the most widely used models is the Hufnagel-Valley (HV) model described by ${ }^{17}$

$$
\begin{aligned}
C_{n}^{2}(h)= & 0.00594(v / 27)^{2}\left(10^{-5} h\right)^{10} \exp (-h / 1000) \\
& +2.7 \times 10^{-16} \exp (-h / 1500)+A \exp (-h / 100),
\end{aligned}
$$

where $h$ is in meters $(\mathrm{m}), v$ is the rms windspeed (pseudowind) in meters per second $(\mathrm{m} / \mathrm{s})$, and $A$ is a nominal value of $C_{n}^{2}(0)$ at the ground in $\mathrm{m}^{-2 / 3}$. In the following analysis, $v=21 \mathrm{~m} / \mathrm{s}, A=1.7 \times 10^{-14}$ or $3 \times 10^{-13} \mathrm{~m}^{-2 / 3}, h_{0}$ is the height above ground of an optical transmitter/receiver, $H$ is the altitude of the satellite (receiver/transmitter), $\zeta$ is the zenith angle, and the total propagation distance to the satellite is $L=\left(H-h_{0}\right) \sec \zeta$. 
For an uplink/downlink communication channel, the spatial-frequency filter function adopted here exhibits the modified form

$$
\begin{aligned}
G\left(\kappa, z ; H, h_{0}\right)= & G_{x}\left(\kappa, z ; H, h_{0}\right)+G_{y}\left(\kappa ; H, h_{0}\right) \\
= & A\left(H, h_{0}\right) \exp \left\{-\int_{0}^{1} D\left[\frac{\kappa \rho_{0}}{\kappa_{x}} w(\tau, z)\right] \mathrm{d} \tau\right\} \\
& +\frac{B\left(H, h_{0}\right) \kappa^{11 / 3}}{\left(\kappa^{2}+\kappa_{y}^{2}\right)^{11 / 6}},
\end{aligned}
$$

where $\kappa_{x}$ and $\kappa_{y}$ are defined similarly to Eqs. (5) and (6), and $A\left(H, h_{0}\right)$ and $B\left(H, h_{0}\right)$ are weighting constants that allow for altitude variations of the structure parameter $C_{n}^{2}(h)$ on the large-scale and small-scale scintillations. Once again, these particular choices of large-scale and small-scale filter functions are based on mathematical convenience rather than on a rigorous physical basis. The function $D(\rho)$ that appears in Eq. (8) is the plane-wave phasestructure function defined by

$$
\begin{aligned}
D(\rho) & =2.914\left(k^{2} \rho^{5 / 3} \sec \zeta\right) \int_{h_{0}}^{H} C_{n}^{2}(h) \mathrm{d} h \\
& =2.914 \mu_{0} k^{2} \rho^{5 / 3} \sec \zeta=2\left(\rho / \rho_{0}\right)^{5 / 3},
\end{aligned}
$$

the function $w(\tau, z)$ depends on propagation distance according to

$w(\tau, z)= \begin{cases}\tau(1-\epsilon z / L), & \tau<z / L, \\ (z / L)(1-\epsilon \tau), & \tau>z / L\end{cases}$

( $\epsilon=0$ for a plane wave and $\epsilon=1$ for a spherical wave), and

$\mu_{0}=\int_{h_{0}}^{H} C_{n}^{2}(h) \mathrm{d} h$.

Although it is not obvious, the low-pass (large-scale) filter function $G_{x}\left(\kappa, z ; H, h_{0}\right)$ in Eq. (8) takes into account that large-scale effects are strongest near the transmitter and that a phase variation at propagation distance $z<L$ induces an amplitude effect at the receiver located at distance $L$ from the transmitter. Moreover, it is chosen in such a way that it leads to results consistent with those developed in Ref. 13 and also agrees with the low-pass filter function in the asymptotic theory for the saturation regime. ${ }^{18-20}$ In this regard, the weighting constant $A\left(H, h_{0}\right)$ reduces to unity in the limiting case of constant $C_{n}^{2}$, and we find the scintillation index in the saturation regime takes the form ${ }^{20}$

$$
\begin{aligned}
\sigma_{I}^{2}(L)= & 1+32 \pi^{2} k^{2} \int_{0}^{L} \int_{0}^{\infty} \kappa \Phi_{n}(\kappa) G_{x}(\kappa, z ; L, 0) \\
& \times \sin ^{2}\left[\frac{\kappa^{2}}{2 k} w(z, z)\right] \mathrm{d} \kappa \mathrm{d} z,
\end{aligned}
$$

where

$$
G_{x}(\kappa, z ; L, 0)=\exp \left\{-\int_{0}^{1} D\left[\frac{L \kappa}{k} w(\tau, z)\right] \mathrm{d} \tau\right\} .
$$

(We should point out that in a recent simulation experiment, Flatte and Gerber ${ }^{21}$ showed that their simulation data for the saturation regime give a somewhat different powerlaw behavior than that predicted by the asymptotic theory. ${ }^{18-20}$ ) Under strong fluctuations, the high-pass filter function $G_{y}\left(\kappa ; H, h_{0}\right)$ depends mostly on small turbulent cells near the receiver and thus exhibits essentially no explicit dependence on the distance variable $z$ along the path We tacitly assume that this is true of the high-pass filter function even in weak fluctuations. Like $A\left(H, h_{0}\right)$, the weighting constant $B\left(H, h_{0}\right)$ in the high-pass filter function also reduces to unity when $C_{n}^{2}$ is constant.

\section{Downlink Channel}

For a downlink path from a satellite, it is well known that the ground-level scintillation near the center of the received wave can be accurately modeled by a plane wave. ${ }^{22}$ Based on weak-scintillation theory and the Kolmogorov spectrum [Eq. (3) with $G(\kappa, z) \equiv 1$ ], the scintillation index for a plane wave is simply the Rytov variance defined by ${ }^{17,22,23}$

$$
\begin{aligned}
\sigma_{1}^{2}= & 2.606 k^{2} \sec \zeta \int_{h_{0}}^{H} C_{n}^{2}(h) \int_{0}^{\infty} \kappa^{-8 / 3} \\
& \times\left\{1-\cos \left[\frac{\left(h-h_{0}\right) \kappa^{2} \sec \zeta}{k}\right]\right\} \mathrm{d} \kappa \mathrm{d} h \\
= & 2.25 \mu_{1} k^{7 / 6}\left(H-h_{0}\right)^{5 / 6} \sec ^{11 / 6} \zeta,
\end{aligned}
$$

where

$\mu_{1}=\int_{h_{0}}^{H} C_{n}^{2}(h)\left(\frac{h-h_{0}}{H-h_{0}}\right)^{5 / 6} \mathrm{~d} h$.

We see from the form of Eq. (15) that the Rytov variance (14) depends mostly on high-altitude turbulence. Hence, we generally expect values of the Rytov variance consistent with weak-fluctuation theory except for the case of large zenith angles. In the following analysis it is sometimes useful to express the Rytov variance (14) in terms of the planewave transverse spatial coherence radius ${ }^{17,23} \rho_{0}$ $=\left(1.45 \mu_{0} k^{2}\right)^{-3 / 5} \cos ^{3 / 5} \zeta$ which leads to

$\sigma_{1}^{2}=1.55\left(\frac{L}{k \rho_{0}^{2}}\right)^{5 / 6} \frac{\mu_{1}}{\mu_{0}}$.

For constant $C_{n}^{2}$, Eqs. (14) and (16) reduce to $\sigma_{1}^{2}$ $=1.23 C_{n}^{2} k^{7 / 6} L^{11 / 6}=0.847\left(L / k \rho_{0}^{2}\right)^{5 / 6}$.

\subsection{Scintillation Index}

By following the approach in Ref. 13, the small-scale logirradiance scintillation takes the form 


$$
\begin{aligned}
\sigma_{\ln y}^{2}= & 2.606 B\left(H, h_{0}\right) k^{2} \sec \zeta \int_{h_{0}}^{H} C_{n}^{2}(h) \int_{0}^{\infty} \frac{\kappa}{\left(\kappa^{2}+\kappa_{y}^{2}\right)^{11 / 6}} \\
& \times\left\{1-\cos \left[\frac{\left(h-h_{0}\right) \kappa^{2} \sec \zeta}{k}\right]\right\} \mathrm{d} \kappa \mathrm{d} h \\
\approx & 1.563\left[B\left(H, h_{0}\right) \mu_{0} k^{7 / 6}\left(H-h_{0}\right)^{5 / 6} \sec ^{11 / 6} \zeta\right] \eta_{y}^{-5 / 6},
\end{aligned}
$$

or, by the use of Eq. (14),

$\sigma_{\ln y}^{2}=0.695 B\left(H, h_{0}\right) \frac{\mu_{0}}{\mu_{1}} \sigma_{1}^{2} \eta_{y}^{-5 / 6}$,

where

$\eta_{y}=\frac{L \kappa_{y}^{2}}{k}=3\left(1+0.69 \sigma_{1}^{12 / 5}\right)$.

The proper choice of weighting constant in Eq. (18) is not clear, but by selecting $B\left(H, h_{0}\right)=1.83\left(\mu_{1} / \mu_{0}\right)$, we note that Eq. (18) reduces to the expression $\sigma_{\ln y}^{2}$ $=1.272 \sigma_{1}^{2} \eta_{y}^{-5 / 6}$ given in Ref. 13. This choice of weighting constant $B\left(H, h_{0}\right)$ also minimizes low-altitude effects in $\sigma_{\ln y}^{2}$ and reduces appropriately to unity in the limiting case of constant $C_{n}^{2}$. Consequently, Eq. (18) becomes

$\sigma_{\ln y}^{2}=\frac{0.51 \sigma_{1}^{2}}{\left(1+0.69 \sigma_{1}^{12 / 5}\right)^{5 / 6}}$.

To find a comparable expression for the large-scale logirradiance variance, we first use Eqs. (9) and (10) with $\epsilon$ $=0$ to simplify the low-pass spatial filter, i.e.,

$$
\begin{aligned}
G_{x}\left(\kappa, z ; H, h_{0}\right) & =A\left(H, h_{0}\right) \exp \left\{-\int_{0}^{1} D\left[\frac{\kappa \rho_{0}}{\kappa_{x}} w(\tau, z)\right] \mathrm{d} \tau\right\} \\
& =A\left(H, h_{0}\right) \exp \left[-2\left(\frac{\kappa}{\kappa_{x}}\right)^{5 / 3} \xi^{5 / 3}\left(1-\frac{5}{8} \xi\right)\right],
\end{aligned}
$$

where $\xi=z / L=\left(h-h_{0}\right) /\left(H-h_{0}\right)$ on a downlink channel. Thus, using the geometrical-optics approximation (i.e., 1 $-\cos x \approx x^{2} / 2$ ), the large-scale log-irradiance variance becomes

$$
\begin{aligned}
\sigma_{\ln x}^{2}= & 2.606 k^{2} \sec \zeta \int_{h_{0}}^{H} C_{n}^{2}(h) \int_{0}^{\infty} \kappa^{-8 / 3} G_{x}\left(\kappa, z ; H, h_{0}\right) \\
& \times\left\{1-\cos \left[\frac{\left(h-h_{0}\right) \kappa^{2} \sec \zeta}{k}\right]\right\} \mathrm{d} \kappa \mathrm{d} h \\
\approx & 1.303 A\left(H, h_{0}\right)\left(H-h_{0}\right)^{2} \sec ^{3} \zeta \\
& \times \int_{h_{0}}^{H} C_{n}^{2}(h) \xi^{2} \int_{0}^{\infty} \kappa^{4 / 3} \exp \left[-2\left(\frac{\kappa}{\kappa_{x}}\right)^{5 / 3} \xi^{5 / 3}\right. \\
& \left.\times\left(1-\frac{5}{8} \xi\right)\right] \mathrm{d} \kappa \mathrm{d} h \\
\approx & 0.263\left[A\left(H, h_{0}\right) \mu_{2} k^{7 / 6}\left(H-h_{0}\right)^{5 / 6} \sec ^{11 / 6} \zeta\right] \eta_{x}^{7 / 6},
\end{aligned}
$$

where $^{13}$

$\mu_{2}=\int_{h_{0}}^{H} \frac{C_{n}(h) \xi^{-1 / 3}}{\left(1-\frac{5}{8} \xi\right)^{7 / 5}} \mathrm{~d} h$,

$\eta_{x}=\frac{L \kappa_{x}^{2}}{k}=\frac{1}{c_{1}+c_{2} \sigma_{1}^{12 / 5}}$.

The scaling constants $c_{1}$ and $c_{2}$ in Eq. (24) will be determined below on the basis of asymptotic behavior under weak and strong fluctuations. In terms of the Rytov variance (14), we can rewrite Eq. (22) as

$\sigma_{\ln x}^{2}=0.116 A\left(H, h_{0}\right)\left(\frac{\mu_{2}}{\mu_{1}}\right) \sigma_{1}^{2} \eta_{x}^{7 / 6}$.

Through arguments analogous to those for the smallscale log-variance, here we set $A\left(H, h_{0}\right)=4.68 \mu_{1} / \mu_{2}$, in which $A\left(H, h_{0}\right)=1$ with constant $C_{n}^{2}$. Also, we set $c_{1}$ $=1.09$, so that Eq. (25) reduces to $\sigma_{\ln x}^{2} \approx 0.49 \sigma_{1}^{2}$ under weak fluctuations $\left(\sigma_{1}^{2} \ll 1\right)$, giving us $\sigma_{\ln x}^{2}+\sigma_{\ln y}^{2}=\sigma_{1}^{2}$. In the saturation regime, we impose the asymptotic result ${ }^{13}$ $A\left(H, h_{0}\right) \eta_{x}^{7 / 6} \approx\left(k \rho_{0}^{2} / L\right)^{7 / 6}=\left[1.7\left(\mu_{1} / \mu_{0}\right)^{6 / 5} \sigma_{1}^{-12 / 5}\right]^{7 / 6}$, where we are recalling $L / k \rho_{0}^{2}=0.59\left(\mu_{0} / \mu_{1}\right)^{6 / 5} \sigma_{1}^{12 / 5}$ from Eq. (16). These results enable us to deduce that

$\eta_{x}=\frac{0.92}{1+2\left(\mu_{1} / \mu_{2}\right)^{6 / 7}\left(\mu_{0} / \mu_{1}\right)^{6 / 5} \sigma_{1}^{12 / 5}}$,

and the large-scale log-irradiance variance (25) becomes

$\sigma_{\ln x}^{2}=\frac{0.49 \sigma_{1}^{2}}{\left[1+2\left(\mu_{1} / \mu_{2}\right)^{6 / 7}\left(\mu_{0} / \mu_{1}\right)^{6 / 5} \sigma_{1}^{12 / 5}\right]^{7 / 6}}$.

It is an interesting observation that the coefficient of $\sigma_{1}^{12 / 5}$ in the denominators of Eqs. (26) and (27) is nearly constant, regardless of the ground-level turbulence strength, upper atmospheric wind speed, and so forth. That is, using the Hufnagle-Valley model (7), we find that (on average)

$2\left(\mu_{1} / \mu_{2}\right)^{6 / 7}\left(\mu_{0} / \mu_{1}\right)^{6 / 5} \approx 1$ to 1.5 . 


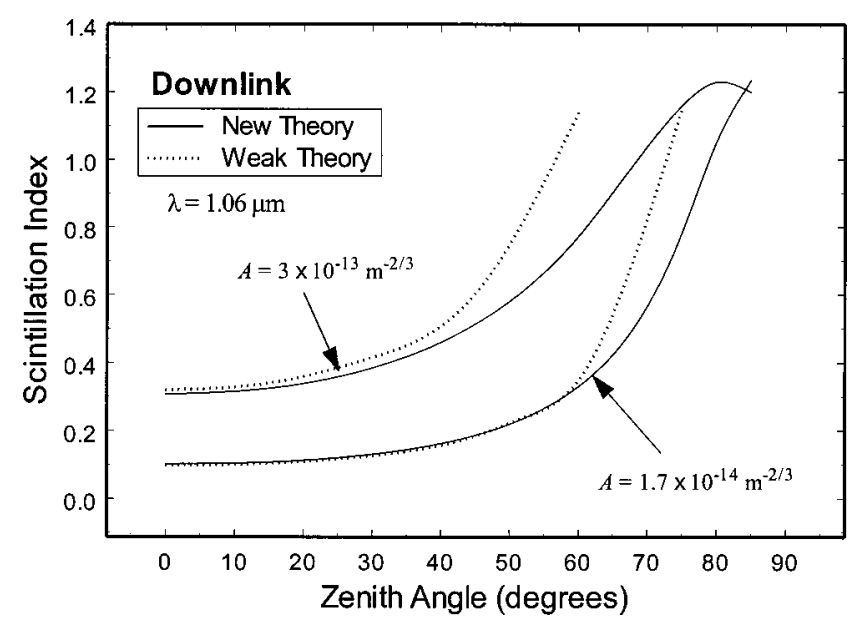

Fig. 1 Scintillation index (solid curves) of a downlink optical wave to a receiver on the ground as a function of zenith angle and two values of ground-level $C_{n}^{2}(0)$. The wavelength is $\lambda=1.06 \mu \mathrm{m}$, and the dotted curves correspond to Eq. (14).

Hence, if we take the nominal value of 1.11 (i.e., the value that arises when $C_{n}^{2}$ is constant) for the expression on the left-hand side in Eq. (28), we arrive at

$\sigma_{\ln x}^{2}=\frac{0.49 \sigma_{1}^{2}}{\left(1+1.11 \sigma_{1}^{12 / 5}\right)^{7 / 6}}$.

Note that Eq. (29) is a minor variation of the large-scale log-amplitude variance derived in Ref. 13.

Finally, we conclude from the above results (2), (20), and (29) that over a downlink optical communication channel in which $H$ is greater than the effective atmospheric layer, the received optical wave is approximately a plane wave in which scintillation index for arbitrary zenith angle and ground-level strength of turbulence is described by

$$
\begin{aligned}
\sigma_{I}^{2}= & \exp \left[\frac{0.49 \sigma_{1}^{2}}{\left(1+1.11 \sigma_{1}^{12 / 5}\right)^{7 / 6}}+\frac{0.51 \sigma_{1}^{2}}{\left(1+0.69 \sigma_{1}^{12 / 5}\right)^{5 / 6}}\right]-1, \\
0 & \leqslant \sigma_{1}^{2}<\infty
\end{aligned}
$$

where the Rytov variance $\sigma_{1}^{2}$ is defined by Eq. (14). At zenith angles exceeding 45 to $60 \mathrm{deg}$ the Rytov variance can be much larger than unity, corresponding to moderate to strong irradiance fluctuations predicted by Eq. (30).

For a satellite positioned in either a low Earth orbit (LEO) or a geostationary orbit (GEO) with receiver on the ground $\left(h_{0}=0\right)$, the scintillation index (30) varies with zenith angle as shown in Fig. 1 for a downlink optical wave with wavelength $\lambda=1.06 \mu \mathrm{m}$. Weak-fluctuation theory (dotted curves) as predicted by Eq. (14) is valid only for zenith angles less than $60 \mathrm{deg}$ when ground-level turbulence is on the order of $A=1.7 \times 10^{-14} \mathrm{~m}^{-2 / 3}$; however, when $A=3 \times 10^{-13} \mathrm{~m}^{-2 / 3}$, weak-fluctuation theory is restricted to zenith angles less than $40 \mathrm{deg}$. For shorter wavelengths, weak-fluctuation theory is limited to even smaller zenith angles.

\subsection{Covariance Function}

Based on results given in Ref. 13, the covariance function of irradiance fluctuations for a downlink propagation path can be expressed as

$$
B_{I}(\rho)=\exp \left[B_{\ln x}(\rho)+B_{\ln y}(\rho)\right]-1,
$$

where $\rho$ denotes the distance between two points on the phase front, and $B_{\ln x}(\rho)$ and $B_{\ln y}(\rho)$ are the log-irradiance covariances of large-scale and small-scale fluctuations, respectively, which reduce to $\sigma_{\ln x}^{2}$ and $\sigma_{\ln y}^{2}$ when $\rho=0$.

Following Ref. 13, the small-scale log-irradiance covariance leads to

$$
\begin{aligned}
B_{\ln y}(\rho)= & 2.606 B\left(H, h_{0}\right) k^{2} \sec \zeta \\
& \times \int_{h_{0}}^{H} C_{n}^{2}(h) \int_{0}^{\infty} \frac{\kappa J_{0}(\kappa \rho)}{\left(\kappa^{2}+\kappa_{y}^{2}\right)^{11 / 6}} \\
& \times\left\{1-\cos \left[\frac{\left(h-h_{0}\right) \kappa^{2} \sec \zeta}{k}\right]\right\} \mathrm{d} \kappa \mathrm{d} h \\
\approx & 1.303\left[B\left(H, h_{0}\right) \mu_{0} k^{7 / 6}\left(H-h_{0}\right)^{5 / 6} \sec ^{11 / 6} \zeta\right] \\
& \times \int_{0}^{\infty} \frac{J_{0}(\rho \sqrt{k \eta / L})}{\left(\eta+\eta_{y}\right)^{11 / 6}} \mathrm{~d} \eta,
\end{aligned}
$$

from which we obtain (similarly to the constant- $C_{n}^{2}$ expression given in Ref. 13)

$B_{\ln y}(\rho)=0.994 \sigma_{\ln y}^{2}\left(\frac{k \rho^{2} \eta_{y}}{L}\right)^{5 / 12} K_{5 / 6}\left[\left(\frac{k \rho^{2} \eta_{y}}{L}\right)^{1 / 2}\right]$,

where $K_{\nu}(x)$ is a Bessel function, $B\left(H, h_{0}\right)$ $=1.83\left(\mu_{1} / \mu_{0}\right), \eta_{y}$ is defined by Eq. (19), and $\sigma_{\ln y}^{2}$ by Eq. (20).

If we use the filter function defined by Eq. (21) for the large-scale fluctuations and impose the geometrical-optics approximation, we obtain the expression

$$
\begin{aligned}
B_{\ln x}(\rho)= & 2.606 k^{2} \sec \zeta \int_{h_{0}}^{H} C_{n}^{2}(h) \int_{0}^{\infty} \kappa^{-8 / 3} J_{0}(\kappa \rho) \\
& \times G_{x}(\kappa, z)\left\{1-\cos \left[\frac{\left(h-h_{0}\right) \kappa^{2} \sec \zeta}{k}\right]\right\} \mathrm{d} \kappa \mathrm{d} h \\
\approx & 1.303 A\left(H, h_{0}\right)\left(H-h_{0}\right)^{2} \sec ^{3} \zeta \\
& \times \int_{h_{0}}^{H} C_{n}^{2}(h) \xi^{2} \int_{0}^{\infty} \kappa^{4 / 3} J_{0}(\kappa \rho) \\
& \times \exp \left[-2\left(\frac{\kappa}{\kappa_{x}}\right)^{5 / 3} \xi^{5 / 3}\left(1-\frac{5}{8} \xi\right)\right] \mathrm{d} \kappa \mathrm{d} h,
\end{aligned}
$$

where $\xi=\left(h-h_{0}\right) /\left(H-h_{0}\right)$ and once again $A\left(H, h_{0}\right)$ $=4.68 \mu_{1} / \mu_{2}$. An exact evaluation of the inner integral in Eq. (34) is unknown. However, through approximation methods it can be shown that

$B_{\ln x}(\rho) \approx \sigma_{\ln x}^{2} \frac{\mu_{2}(\rho)}{\mu_{2}(0)}$ 


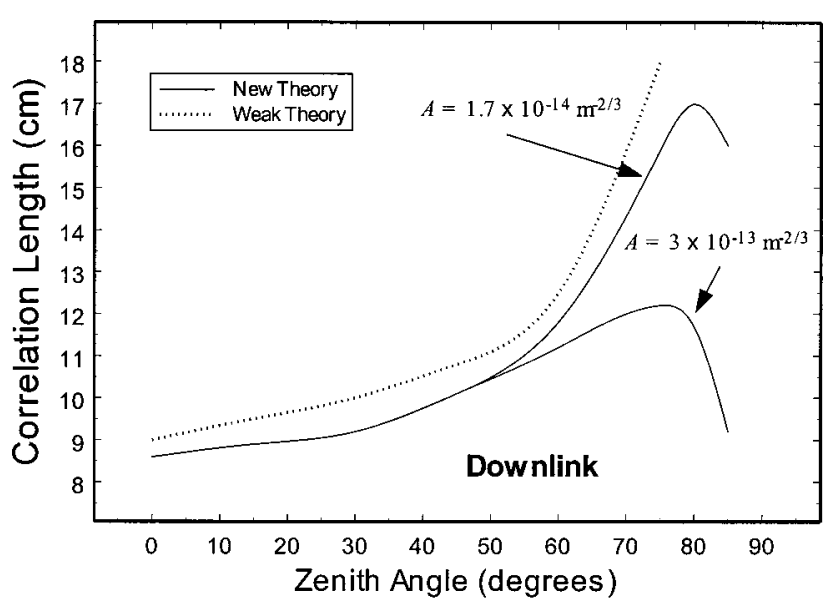

Fig. 2 Correlation length as determined by the 1/e point of the covariance function (37), scaled by the scintillation index (30), and shown as a function of zenith angle for a downlink optical wave from a satellite in orbit. The dotted curve is based on standard Rytov theory valid under weak-fluctuation conditions.

where

$$
\begin{aligned}
\mu_{2}(\rho)= & \int_{h_{0}}^{H} \frac{C_{n}^{2}(h) \xi^{-1 / 3}}{\left(1-\frac{5}{8} \xi\right)^{7 / 5}} \\
& \times{ }_{1} F_{1}\left[\frac{7}{5} ; 1 ; \frac{-k \rho^{2} \eta_{x}}{8 L \xi^{5 / 3}\left(1-\frac{5}{8} \xi\right)}\right] \mathrm{d} h,
\end{aligned}
$$

$\eta_{x}=0.92 /\left(1+1.11 \sigma_{1}^{12 / 5}\right), \sigma_{\ln x}^{2}$ is defined by Eq. (29), and ${ }_{1} F_{1}(a ; c ; x)$ is a confluent hypergeometric function. ${ }^{24} \mathrm{Com}-$ bining the results of Eqs. (33) and (35), the covariance function (31) can be written as

$$
\begin{aligned}
B_{I}(\rho)= & \exp \left\{\sigma_{\ln x}^{2} \frac{\mu_{2}(\rho)}{\mu_{2}(0)}+0.994 \sigma_{\ln y}^{2}\left(\frac{k \rho^{2} \eta_{y}}{L}\right)^{5 / 12}\right. \\
& \left.\times K_{5 / 6}\left[\left(\frac{k \rho^{2} \eta_{y}}{L}\right)^{1 / 2}\right]\right\}-1 .
\end{aligned}
$$

In Fig. 2 we show the implied correlation length (solid curves) of a ground-based receiver associated with a downlink communication path from a satellite in orbit. The correlation length $\rho_{c}$ is defined by the $1 / e$ point of the covariance function (37) scaled by the scintillation index (30). Notice that the two dotted curves, corresponding to $A$ $=1.7 \times 10^{-14} \mathrm{~m}^{-2 / 3}$ and $A=3 \times 10^{-13} \mathrm{~m}^{-2 / 3}$, coincide under weak-fluctuation conditions (zenith angles not exceeding $50 \mathrm{deg}$ ). In fact, we found that the correlation length $\rho_{c}$ for such conditions is roughly determined by the empirical relation

$\rho_{c} \approx\left(\frac{45 \times 10^{3} \sec \zeta}{k}\right)^{1 / 2}, \quad \sigma_{1}^{2} \ll 1$.

Also shown in Fig. 2 for comparison is the implied correlation length (dotted curve) derived from standard Rytov weak-fluctuation theory, based on the zero-crossing point of the normalized covariance function. Clearly, the new results are in reasonably good agreement with conventional weak-fluctuation theory for zenith angles less than $50 \mathrm{deg}$.

Under strong fluctuations (zenith angles greater than 50 deg), the implied correlation length illustrated in Fig. 2 is more sensitive to ground level-turbulence. In the saturation regime, for example, the correlation length is roughly characterized by

$\rho_{c} \approx 0.53\left(\mu_{0} / \mu_{1}\right)^{3 / 5} \rho_{0}, \quad \sigma_{1}^{2}>10$.

Last, we caution the reader that the results shown in Fig. 2 for the present theory are based on a LEO satellite using the 1/e point of the normalized covariance function. For a GEO satellite, the same results are deduced from the halfpower point of the normalized correlation function rather than the 1/e point. This particular sensitivity to the height of the satellite is a peculiarity of the current model and does not exist in conventional weak-fluctuation models.

\section{Uplink Channel}

For an uplink optical wave model, we consider the case of a diverged beam that acts like a point source or spherical wave. Under weak scintillations, the normalized variance of irradiance is described by ${ }^{8}$

$\sigma_{2}^{2}=2.25 \mu_{3} k^{7 / 6}\left(H-h_{0}\right)^{5 / 6} \sec ^{11 / 6} \zeta$,

where

$\mu_{3}=\int_{h_{0}}^{H} C_{n}^{2}(h) \xi^{5 / 6}(1-\xi)^{5 / 6} \mathrm{~d} h$,

and, for an uplink path, $\xi=1-\left(h-h_{0}\right) /\left(H-h_{0}\right)$. Note that the functional form of the integral in Eq. (41) suggests that it can be closely approximated by

$\mu_{3} \approx \mu_{1} \int_{h_{0}}^{H} C_{n}^{2}(h)\left(\frac{h-h_{0}}{H-h_{0}}\right)^{5 / 6} \mathrm{~d} h$.

Consequently, the scintillation index (40) for an uplink spherical wave in the weak-fluctuation regime is equivalent to the Rytov variance $\sigma_{1}^{2}$ for a downlink plane wave given by Eq. (14). ${ }^{8}$ Thus, $\sigma_{2}^{2}$ is considered a measure of the strength of turbulence when it exceeds unity, similar to our interpretation of $\sigma_{1}^{2}$. However, in the limiting case of a horizontal path with constant $C_{n}^{2}$, we find that $\sigma_{2}^{2}$ is equivalent to the conventional spherical-wave result $\sigma_{2}^{2}=\beta_{0}^{2}$ $=0.4 \sigma_{1}^{2}$.

\subsection{Scintillation Index}

To begin our analysis for an uplink channel, we follow the downlink analysis for the small-scale log-irradiance variance, which leads to 


$$
\begin{aligned}
\sigma_{\ln y}^{2}= & 2.606 B\left(H, h_{0}\right) k^{2} \sec \zeta \int_{h_{0}}^{H} C_{n}^{2}(h) \int_{0}^{\infty} \frac{\kappa}{\left(\kappa^{2}+\kappa_{y}^{2}\right)^{11 / 6}} \\
& \times\left\{1-\cos \left[\frac{L \kappa^{2}}{k} \xi(1-\xi)\right]\right\} \mathrm{d} \kappa \mathrm{d} h \\
\approx & 1.563\left[B\left(H, h_{0}\right) \mu_{0} k^{7 / 6}\left(H-h_{0}\right)^{5 / 6} \sec ^{11 / 6} \zeta\right] \eta_{y}^{-5 / 6},
\end{aligned}
$$

or, equivalently,

$\sigma_{\ln y}^{2}=0.695 B\left(H, h_{0}\right)\left(\frac{\mu_{0}}{\mu_{3}}\right) \sigma_{2}^{2} \eta_{y}^{-5 / 6}$,

where the weighting constant $B\left(H, h_{0}\right)$ and nondimensional parameter $\eta_{y}$ are not the same as those for the downlink case. In particular, to ensure that the weighting constant in Eq. (44) reduces to unity when $C_{n}^{2}$ is constant, we select $B\left(H, h_{0}\right)=4.535 \mu_{3} / \mu_{0}$. For the nondimensional parameter $\eta_{y}$, we set

$\eta_{y}=8.92\left(1+0.69 \sigma_{2}^{12 / 5}\right)$,

which reduces Eq. (44) to a form comparable with Eq. (20), viz.,

$\sigma_{\ln y}^{2}=\frac{0.51 \sigma_{2}^{2}}{\left(1+0.69 \sigma_{2}^{12 / 5}\right)^{5 / 6}}$.

For an uplink spherical wave, we must recalculate the large-scale filter function comparable to Eq. (21). In this case we set $\epsilon=1$ in Eq. (10) and obtain

$$
\begin{aligned}
G_{x}\left(\kappa, z ; H, h_{0}\right) & =A\left(H, h_{0}\right) \exp \left\{-\int_{0}^{1} D\left[\frac{\kappa \rho_{0}}{\kappa_{x}} w(\tau, z)\right] \mathrm{d} \tau\right\} \\
& =A\left(H, h_{0}\right) \exp \left[-\frac{3}{4}\left(\frac{\kappa}{\kappa_{x}}\right)^{5 / 3} \xi^{5 / 3}(1-\xi)^{5 / 3}\right],
\end{aligned}
$$

where $\kappa_{x}$ is the low-pass cutoff spatial frequency and $A\left(H, h_{0}\right)$ is the weighting constant for the uplink path. From Eq. (47), we deduce that

$$
\begin{aligned}
\sigma_{\ln x}^{2}= & \left(2.606 k^{2} \sec \zeta\right) \int_{h_{0}}^{H} C_{n}^{2}(h) \int_{0}^{\infty} \kappa^{-8 / 3} G_{x}\left(\kappa, z ; H, h_{0}\right) \\
& \times\left\{1-\cos \left[\frac{L \kappa^{2}}{k} \xi(1-\xi)\right]\right\} \mathrm{d} k \mathrm{~d} h \\
\approx & 1.303 A\left(H, h_{0}\right)\left(H-h_{0}\right)^{2} \sec ^{3} \zeta \\
& \times \int_{h_{0}}^{H} C_{n}^{2}(h) \xi^{2}(1-\xi)^{2} \int_{0}^{\infty} \kappa^{4 / 3} \\
& \times \exp \left[-\frac{3}{4}\left(\frac{\kappa}{\kappa_{x}}\right)^{5 / 3} \xi^{5 / 3}(1-\xi)^{5 / 3}\right] \mathrm{d} \kappa \mathrm{d} h \\
\approx & {\left[1.04 A\left(H, h_{0}\right) \mu_{4} k^{7 / 6}\left(H-h_{0}\right)^{5 / 6} \sec ^{11 / 6} \zeta\right] \eta_{x}^{7 / 6} }
\end{aligned}
$$

or, using Eq. (40),

$\sigma_{\ln x}^{2}=0.462 A\left(H, h_{0}\right)\left(\frac{\mu_{4}}{\mu_{3}}\right) \sigma_{2}^{2} \eta_{x}^{7 / 6}$,

where

$\mu_{4}=\int_{h_{0}}^{H} C_{n}^{2}(h) \xi^{-1 / 3}(1-\xi)^{-1 / 3} \mathrm{~d} h$.

Here, we set $A\left(H, h_{0}\right)=9.31 \mu_{3} / \mu_{4}$ and, by assuming the parameter $\eta_{x}$ is defined analogously to Eq. (24) and enforcing the weak-fluctuation asymptotic result $\sigma_{\ln x}^{2} \approx 0.49 \sigma_{2}^{2}$, we find that $c_{1}=6.41$. Also, in the saturation regime, we have $A\left(H, h_{0}\right) \eta_{x}^{7 / 6} \approx\left(k \rho_{0}^{2} / L\right)^{7 / 6}=\left[1.7\left(\mu_{3} / \mu_{0}\right)^{6 / 5} \sigma_{2}^{-12 / 5}\right]^{7 / 6}$, which leads to

$\eta_{x}=\frac{0.156}{1+0.62\left(\mu_{3} / \mu_{4}\right)^{6 / 7}\left(\mu_{0} / \mu_{3}\right)^{6 / 5} \sigma_{2}^{12 / 5}}$.

Thus, the large-scale log-irradiance variance (49) becomes

$\sigma_{\ln x}^{2}=\frac{0.49 \sigma_{2}^{2}}{\left[1+0.62\left(\mu_{3} / \mu_{4}\right)^{6 / 7}\left(\mu_{0} / \mu_{3}\right)^{6 / 5} \sigma_{2}^{12 / 5}\right]^{7 / 6}}$.

Because $\mu_{3} \approx \mu_{1}$ and $\mu_{4} \approx \mu_{2}$, it follows from Eq. (28) that Eq. (52) can be replaced by

$\sigma_{\ln x}^{2}=\frac{0.49 \sigma_{2}^{2}}{\left(1+0.56 \sigma_{2}^{12 / 5}\right)^{7 / 6}}$.

From these results we see that the scintillation index for an uplink spherical wave under weak to strong fluctuations is given by

$$
\begin{aligned}
\sigma_{1}^{2}= & \exp \left[\frac{0.49 \sigma_{2}^{2}}{\left(1+0.56 \sigma_{2}^{12 / 5}\right)^{7 / 6}}+\frac{0.51 \sigma_{2}^{2}}{\left(1+0.69 \sigma_{2}^{12 / 5}\right)^{5 / 6}}\right]-1, \\
0 & \leqslant \sigma_{2}^{2}<\infty
\end{aligned}
$$

where $\sigma_{2}^{2}$ is defined by Eq. (40).

We note that Eq. (54) differs from Eq. (30) for the downlink case under moderate to strong fluctuations, but agrees with Eq. (30) for weak fluctuations. This difference in behavior at large zenith angles is illustrated in Fig. 3 for the same ground-level values of the structure parameter $A$ as considered in Fig. 1. Notice that for zenith angles exceeding $60 \mathrm{deg}$ the scintillation index predicted by Eq. (54) is somewhat greater (by more than $30 \%$ ) than that shown in Fig. 1 for the downlink case.

\subsection{Covariance Function}

Although the scintillation index for an uplink spherical wave is similar to that for a downlink plane wave, the same is not true of the corresponding covariance function. In particular, for a spherical wave on an uplink path the smallscale log-irradiance covariance is defined by 


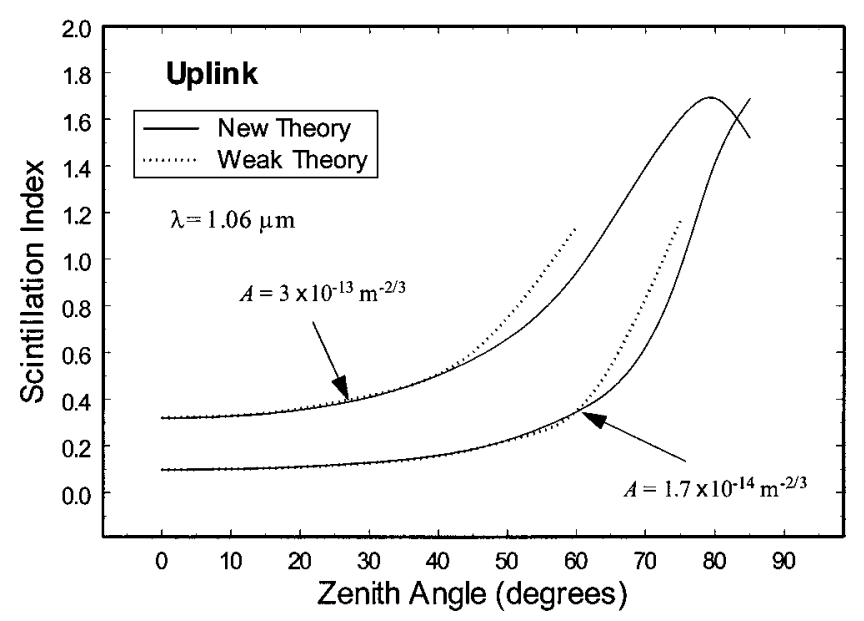

Fig. 3 Scintillation index (solid curves) of an uplink optical wave to a receiver on the ground as a function of zenith angle and two values of ground-level $C_{n}^{2}(0)$. The wavelength is $\lambda=1.06 \mu \mathrm{m}$, and the dotted curves correspond to Eq. (14).

$$
\begin{aligned}
B_{\ln y}(\rho)= & 2.606 B\left(H, h_{0}\right) k^{2} \sec \zeta \\
& \times \int_{h_{0}}^{H} C_{n}^{2}(h) \int_{0}^{\infty} \frac{\kappa}{\left(\kappa^{2}+\kappa_{y}^{2}\right)^{11 / 6}} J_{0}[\kappa \rho(1-\xi)] \\
& \times\left\{1-\cos \left[\frac{L \kappa^{2}}{k} \xi(1-\xi)\right]\right\} \mathrm{d} \kappa \mathrm{d} h \\
\approx & 1.303 B\left(H, h_{0}\right) k^{7 / 6}\left(H-h_{0}\right)^{5 / 6} \sec ^{11 / 6} \zeta \\
& \times \int_{h_{0}}^{H} C_{n}^{2}(h) \int_{0}^{\infty} \frac{J_{0}[\rho(1-\xi) \sqrt{k \eta / L}]}{\left(\eta+\eta_{y}\right)^{11 / 6}} \mathrm{~d} \eta \mathrm{d} h,
\end{aligned}
$$

where $\quad \xi=1-\left(h-h_{0}\right) /\left(H-h_{0}\right) \quad$ and $B\left(H, h_{0}\right)$ $=4.535 \mu_{3} / \mu_{0}$. The evaluation of these integrals yields

$B_{\ln y}(\rho) \approx 0.944 \sigma_{\ln y}^{2}\left(\frac{k \rho^{2} \eta_{y}}{L}\right)^{5 / 12} \frac{\mu_{5}(\rho)}{\mu_{0}}$,

where $\sigma_{\ln y}^{2}$ is defined by Eq. (46), $\eta_{y}$ is defined by Eq. (45), and

$$
\begin{aligned}
\mu_{5}(\rho)= & \int_{h_{0}}^{H} C_{n}^{2}(h)\left(\frac{h-h_{0}}{H-h_{0}}\right)^{5 / 6} \\
& \times K_{5 / 6}\left[\left(\frac{h-h_{0}}{H-h_{0}}\right) \sqrt{\frac{k \rho^{2} \eta_{y}}{L}}\right] \mathrm{d} h .
\end{aligned}
$$

In a similar manner, the large-scale log-irradiance covariance under the geometrical optics approximation leads to

$$
\begin{aligned}
B_{\ln x}(\rho) \approx & 1.303 A\left(H, h_{0}\right)\left(H-h_{0}\right)^{2} \sec ^{3} \zeta \\
& \times \int_{h_{0}}^{H} C_{n}^{2}(h) \xi^{2}(1-\xi)^{2} \int_{0}^{\infty} \kappa^{4 / 3} \\
& \times \exp \left[-\frac{3}{4}\left(\frac{\kappa}{\kappa_{x}}\right)^{5 / 3} \xi^{5 / 3}(1-\xi)^{5 / 3}\right] \\
& \times J_{0}[\kappa \rho(1-\xi)] \mathrm{d} \kappa \mathrm{d} h \\
\approx & \sigma_{\ln x}^{2} \frac{\mu_{4}(\rho)}{\mu_{4}}
\end{aligned}
$$

where $A\left(H, h_{0}\right)=9.31 \mu_{3} / \mu_{4}$ and

$$
\begin{aligned}
\mu_{4}(\rho)= & \int_{h_{0}}^{H} C_{n}^{2}(h) \xi^{-1 / 3}(1-\xi)^{-1 / 3} \\
& \times{ }_{1} F_{1}\left[\frac{7}{5} ; 1 ; \frac{-k \rho^{2} \eta_{x}(1-\xi)^{1 / 3}}{3 L \xi^{5 / 3}}\right] \mathrm{d} h .
\end{aligned}
$$

The nondimensional parameter $\eta_{x}=0.156 /\left(1+0.56 \sigma_{2}^{12 / 5}\right)$, and the large-scale $\log$-irradiance scintillation $\sigma_{\ln x}^{2}$ is defined by Eq. (53). By summing the results (56) and (58), we have the irradiance covariance function for an uplink spherical wave given by

$$
\begin{aligned}
B_{I}(\rho)= & \exp \left[\sigma_{\ln x}^{2} \frac{\mu_{4}(\rho)}{\mu_{4}}+0.944 \sigma_{\ln y}^{2}\left(\frac{k \rho^{2} \eta_{y}}{L}\right)^{5 / 12} \frac{\mu_{5}(\rho)}{\mu_{0}}\right] \\
& -1
\end{aligned}
$$

Unlike the downlink path, here we find the correlation length at a satellite in either LEO or GEO to be on the order of a hundred meters or more, much greater than the probable size of any satellite.

\section{Summary}

In this paper we have extended the heuristic theory developed by Andrews et al. ${ }^{13}$ for moderate to strong optical scintillation along a horizontal path with constant $C_{n}^{2}$ to uplink/downlink propagation paths applicable to satellite communication systems. The scintillation model developed for a downlink path from a satellite has the same functional form as that for horizontal-path propagation of an infinite plane wave, except here the Rytov variance is described by Eq. (14). Under weak fluctuations (small zenith angles), the same model is also valid for an uplink diverged or spherical wave; however, our results suggest that greater scintillations occur for an uplink path with large zenith angles than for the downlink case. In addition to the scintillation model, we have also developed expressions for the corresponding covariance function of irradiance for uplink/downlink paths. In all cases, the new results are in good agreement with conventional weak-scintillation theory for zenith angles less than 45 to $60 \mathrm{deg}$, but departures from weakscintillation theory are predicted by the new models for larger zenith angles. 


\section{Acknowledgment}

Funding for this work was partly provided by the Ballistic Missile Defense Organization's Innovative Science and Technology Directorate and administrated by the Space and Naval Warfare Systems Center, San Diego, CA, under contract No. N66001-97-C-8644.

\section{References}

1. S. G. Lambert and W. L. Casey, Laser Communications in Space, Artech House, Boston (1995)

2. B. L. Edelson and G. Hyde, "A report of the IEEE-USA Aerospace Policy Committee on Laser Satellite Communications, Programs Technology and Applications," (1996).

3. D. L. Fried, "Scintillation of a ground-to-space laser illuminator,"' $J$. Opt. Soc. Am. 57, 980-983 (1967).

4. P. O. Minott, "Scintillation in an earth-to-space propagation path," $J$. Opt. Soc. Am. 62, 885-888 (1972).

5. P. J. Titterton, "Scintillation and transmitter-aperture averaging over vertical paths," J. Opt. Soc. Am. 63, 439-444 (1972).

6. J. L. Bufton, R. S. Tyler, and L. S. Taylor, "Scintillation statistics caused by atmospheric turbulence and speckle in satellite laser ranging,"' Appl. Opt. 16, 2408-2413 (1977).

7. J. L. Bufton, "Scintillation statistics measured in an earth-space-earth retro-reflected link," Appl. Opt. 16, 2654-2660 (1977).

8. H. T. Yura and W. G. McKinley, "Optical scintillation statistics for IR ground-to-space laser communication systems," Appl. Opt. 22, 3353-3358 (1983)

9. P. A. Lightsey, "Scintillation in ground-to-space and retroreflected laser beams,"' Opt. Eng. 33(8), 2535-2543 (1994).

10. L. C. Andrews, R. L. Phillips, and P. T. Yu, "Optical scintillations and fade statistics for a satellite-communication system," Appl. Opt. 34, 7742-7751 (1995); Errata, 36, 6068 (1997).

11. J. D. Shelton, "Turbulence-induced scintillation on Gaussian-beam waves: theoretical predictions and observations from a laserilluminated satellite," J. Opt. Soc. Am. A 12, 2172-2181 (1995).

12. R. K. Tyson, "Adaptive optics and ground-to-space laser communications," Appl. Opt. 35, 3640-3646 (1996).

13. L. C. Andrews, R. L. Phillips, C. Y. Hopen, and M. A. Al-Habash, "Theory of optical scintillation," J. Opt. Soc. Am. A 16, 1417-1429 (1999).

14. E. Jakeman and P. N. Pusey, "A model for non-Rayleigh sea echo," IEEE Trans. Antennas Propag. AP-24, 806-814 (1976).

15. L. C. Andrews and R. L. Phillips, "Mathematical genesis of the $I-K$ distribution for random optical fields," J. Opt. Soc. Am. A 3, 19121919 (1986)

16. J. H. Churnside and S. F. Clifford, "Log-normal Rician probabilitydensity function of optical scintillations in the turbulent atmosphere,' J. Opt. Soc. Am. A 4, 1923-1930 (1987).

17. R. R. Beland, "Propagation through atmospheric optical turbulence,", Chap. 2 in The Infrared and ElectroOptical Systems Handbook, F. G. Smith, Ed., SPIE Optical Engineering Press, Bellingham, WA (1993).

18. K. S. Gochelashvili and V. I. Shishov, "Saturated fluctuations in the laser radiation intensity in a turbulent medium," Sov. Phys. JETP 39, 605-609 (1974).

19. R. L. Fante, "Inner-scale size effect on the scintillations of light in the turbulent atmosphere," J. Opt. Soc. Am. 73, 277-281 (1983).

20. R. G. Frehlich, "Intensity covariance of a point source in a random medium with a Kolmogorov spectrum and an inner scale of turbulence,"' J. Opt. Soc. Am. A 4, 360-366 (1987); Errata, 4, 1324 (1987).

21. S. M. Flatte and J. S. Gerber, "Irradiance-variance behavior by numerical simulation for plane-wave and spherical-wave optical propagation through strong turbulence," J. Opt. Soc. Am. A 17, 1092-1097 (2000).
22. V. I. Tatarskii, Wave Propagation in a Turbulent Medium (transl. by R. A. Silverman), McGraw-Hill, New York (1961).

23. L. C. Andrews and R. L. Phillips, Laser Beam Propagation through Random Media, SPIE Optical Engineering Press, Bellingham, WA (1998).

24. L. C. Andrews, Special Functions of Mathematics for Engineers, 2nd ed., SPIE Optical Engineering Press, Bellingham, WA, and Oxford University Press, Oxford (1998) [previously published as 2nd ed. by McGraw-Hill, New York (1992)].

Larry C. Andrews is a professor of mathematics at the University of Central Florida and a member of the Department of Electrical and Computer Engineering. Dr. Andrews is also an associate member of the School of Optics/CREOL and an associate member of the Florida Space Institute (FSI). Previously, he held a faculty position at Tri-State University and was a staff mathematician with the Magnavox Company antisubmarine warfare operation. He received a doctoral degree in theoretical mechanics in 1970 from Michigan State University. Dr. Andrews has been an active researcher in optical wave propagation through random media for 20 years and is the author of several textbooks on differential equations, boundaryvalue problems, special functions, and integral transforms. Along with wave propagation through random media, his research interests include special functions, random variables, atmospheric turbulence, and signal processing.

Ronald L. Phillips is director of the Florida Space Institute (FSI) and a professor in the Department of Electrical and Computer Engineering at the University of Central Florida. Dr. Phillips is also a member of the Department of Mathematics and an associate member of the School of Optics/CREOL. He has held positions on the faculties at Arizona State University and the University of California, San Diego. He received a doctoral degree in electrical engineering in 1970 from Arizona State University. Dr. Phillips has been an active researcher in wave propagation through random media for more than 25 years. He was awarded a Senior NATO Postdoctoral Fellow in 1977 and the American Society for Engineering Education 1983 Medal for outstanding contributions in research. In addition to optical wave propagation, his research interests include optical communications and imaging through atmospheric turbulence.

Cynthia Young Hopen is an assistant professor of mathematics and a research scientist for the Florida Space Institute at the University of Central Florida. She holds a BA in mathematics education from the University of North Carolina, an MS in mathematical science from the University of Central Florida, and an MS in electrical engineering and $\mathrm{PhD}$ in applied mathematics from the University of Washington, Seattle. Dr. Hopen was awarded a National Physical Science Consortium doctoral fellowship with Kennedy Space Center (KSC) as her sponsor. She has worked with chemists in the Material Science Division and physicists in the Optics Lab at KSC. In 1997 she joined the faculty at FSI/UCF, where she presently is doing research in the area of laser communications and laser radar. She has worked on laser satellite communication projects through the Navy and laser radar projects for the Air Force. She has published fifteen papers in the area of laser propagation through atmospheric turbulence. 